\title{
1 Ecological dynamics of the gut microbiome in response to dietary fiber
}

2 Hongbin Liu ${ }^{1, *}$, Chen Liao ${ }^{2, *}$, Jinhui Tang ${ }^{1,}$, Junyu Chen ${ }^{1,}$, Chaobi Lei ${ }^{1,}$, Linggang Zheng ${ }^{1,}$, Lu Wu ${ }^{1}$, 3 Chenhong Zhang ${ }^{3}$, Yang-Yu Liu ${ }^{4}$, Joao Xavier ${ }^{2}$, Lei Dai ${ }^{1, \#}$

41 CAS Key Laboratory of Quantitative Engineering Biology, Shenzhen Institute of Synthetic Biology, 5 Shenzhen Institutes of Advanced Technology, Chinese Academy of Sciences, Shenzhen 518055, China

62 Program for Computational and Systems Biology, Memorial Sloan-Kettering Cancer Center, New York, 7 NY, USA

83 State Key Laboratory of Microbial Metabolism, Ministry of Education, Laboratory of Systems 9 Biomedicine, Shanghai Jiao Tong University, Shanghai, China

104 Channing Division of Network Medicine, Department of Medicine, Brigham and Women's Hospital and 11 Harvard Medical School, Boston, Massachusetts 02115, USA

$12 *$ : These authors contributed equally to this work

13 \#: Correspondence: lei.dai@ siat.ac.cn

\section{Abstract}

16 Dietary fibers are generally thought to benefit intestinal health. Their impacts on the composition and 17 metabolic function of the gut microbiome, however, vary greatly across individuals. Previous research 18 showed that each individual's response to fibers depends on their baseline gut microbiome, but the ecology 19 driving microbiota remodeling during fiber intake remained unclear. Here, we studied the long-term 20 dynamics of gut microbiome and short-chain fatty acids (SCFAs) in isogenic mice with distinct microbiota 21 baselines fed with the fermentable fiber inulin compared to the non-fermentable fiber cellulose. We found 22 that inulin produced generally rapid response followed by gradual stabilization to new equilibria, and those 23 dynamics were baseline-dependent. We parameterized an ecology model from the timeseries data, which 24 revealed a group of bacteria whose growth significantly increases in response to inulin. and whose baseline 25 abundance and interspecies competition explains the baseline-dependence of microbiome density and 26 community composition dynamics. Fecal levels of of SCFAs, such as propionate, is associated with the 27 abundance of inulin responders, yet inter-individual variation of gut microbiome impedes the prediction of 28 SCFAs by machine learning models. Finally, we showed that our methods and major findings are 29 generalizable to dietary resistant starch. This study emphasizes the importance of ecological modeling to 30 understand microbiome responses to dietary changes and the need for personalized interventions. 


\section{Introduction}

33 Fermentable dietary fibers, such as inulin and resistant starch, are edible carbohydrate polymers that escape

34 digestion by host enzymes in the upper gut and are fermented by gut microbiota in the cecum and colon.

35 The major products from the microbial fermentative activity in the large intestine are short-chain fatty acids

36 (SCFAs) - mainly acetate, propionate and butyrate — which have broad impacts on intestinal health and

37 immunity [1-3]. Impaired SCFAs production has been associated with multiple dieseases [4, 5]. Dietary

38 fibers can selectively enrich beneficial gut bacteria [6] and could be administered as "pre-biotic" therapies to restore intestinal gut microbiota and elevate SCFA levels [7, 8].

Previous work showed that dietary fibers can cause rapid changes in microbiota composition and biomass $[9,10]$. But the ability of fibers to increase SCFA production varies across individuals [11-14]. For example, Baxter et al. showed that resistant starch was able to promote butyrate production in only $63 \%$ participants [12]. The individualized response can arise from a combination of factors such as host genetics and diet history. But the baseline gut microbiota is also a critical factor $[14,15]$. The feces of some healthy human donors fail to ferment resistant starch, which can be restored by co-incubation with $R$. brommi, a well-known degrader of resistant starch [16]. Person-to-person variation in the bacterial and metabolomic composition of gut microbiome [17] can further impact biological variables such as body mass index [18] and glucose tolerance [19] of the human host.

Dietary fibers select from the pool of baseline community for microbial taxa that can use fibers as substrates for growth, and these responders could further impact the entire gut microbial community through a complex ecological network [20] (Fig. 1A). The primary users of dietary fibers are relatively few in low-fiber diet, but they can rapidly expand and dominate the gut microbiota after substantial induction [21]. Production of some SCFAs, especially butyrate, involves cross-feeding cooperations among specialized gut bacteria. By hydrolyzing complex polysaccharide fibers, primary degraders release into the gut partially breakdown products (e.g., mono- and oligo-saccharides) and fermentation metabolites (e.g., pyruvate), which can respectively benefit the secondary fiber degraders and SCFAs producers [22, 23]. Despite increasing interests in microbiota ecology and targeted modulation [24-27], a system-level, quantitative understanding of the ecological dyanmics of gut microbiome under dietary interventions and the dependence on the baseline community composition is still lacking.

In this study, we profile longitudinally the gut microbiota of mice to study the ecological basis for the baseline-dependent dynamical response to dietary fibers. We use the time series data to infer the

62 ecological network that explains why the microbiota fiber responses vary with their baseline composition.

63 We find that the growth rates and ecological interactions of key responders-bacteria with known ability

64 to benefit from fibers - drive the major shifts in the gut microbiota composition. And we identify the set of

65 putative fiber-degrading bacteria whose baseline abundances predict individual responses in bacterial 
absolute abundance and SCFA. This study provides a framework to identify the ecological drivers of microbiota response to dietary interventions, which is critical for understanding the individualized responses of gut microbiota and the design of targeted modulations.

\section{Results}

\section{Isogenic mice from different origins vary in their baseline gut microbiota}

72 We used age- and gender-matched isogenic mice that harbor distinct baseline gut microbiota composition

73 to study the dynamical response to dietary interventions and the inter-individual variation in ecological

74 dynamics [28]. To ensure the distinctness of their baseline gut microbiomes, mice were purchased from

75 four commercial vendors (labeled as Beijing, Guangdong, Hunan, Shanghai, see Methods), i.e. independent

76 breeder sources. All mice were fed with cellulose-based diet 7 days prior to dietary fiber intervention. We

77 monitored temporal shifts in the absolute abundance (by quantitative PCR) and community composition of

78 gut bacteria (by 16S rRNA amplicon sequencing and shotgun metagenomics sequencing), SCFAs 79 concentration (by targeted metabolomics), as well as physiological changes following the intervention of

80 fermentable dietary fibers and cellulose (control group) (Fig. 1B). The two dietary fibers used in this study,

81 inulin and resistant starch from maize, are able to be degraded by gut bacteria in the cecum/colon [29, 30]

82 and to stimulate the production of SCFAs [8, 12].

83 Consistent with previous studies [31,32], these mice grown in vendor-specific housing and feeding 84 conditions can be naturally divided by vendor sources into groups with distinct microbiota composition 85 after 7 days of acclimatization. Principal coordinate analysis based on the robust Aitchison distance [33] 86 shows that the baseline compositions of those mice can be naturally clustered by vendors (Adonis, $P<$ 87 0.001) and are characterized by distinct bacterial taxa (Fig. 1C, Fig. S1A). For example, Shanghai mice 88 have low relative abundances of several commensal polysaccharide-degrading bacteria such as 89 Muribaculaceae and Rikenellaceae [34, 35]. The profound inter-vendor differences are also noticeable at 90 the level of presence and absence of bacteria: 65\% taxa were entirely absent in at least one vendor and 91 only $\sim 10 \%$ bacterial taxa were present in all mice (and thus all vendors) (Fig. 1D). Interestingly, the 92 prevalence and abundance of bacterial taxa in these baseline samples exihibit a strong positive linear 93 relationship in logarithmic scale (Fig. S1B). Due to the high between-vendor variation, mice from the same 94 vendor can be effectively considered as independent biological replicates for each baseline microbiota 95 composition. Throughout the observation of our experiment, the body weight of mice gradually increased 96 over time, but the gain in body weight is generally insignificant between the inulin treatment group and the 97 cellulose control group (Fig. S2A). Among the different experimental groups, there were no obvious 98 difference in food intake and fecal weight (Fig. S2). 


\section{Baseline-dependent microbiota dynamics in response to inulin}

101 Inulin intervention rapidly promoted the absolute abundance of gut bacteria on the time scale of days, except

102 for Shanghai mice (Fig. 2A). More interestingly, inulin induced a two-phase dynamical response in the gut

103 microbiota diversity (Fig. 2B), which dropped rapidly in the short-term and recovered gradually in the long-

104 term. The initial loss of diversity is primarily due to the changes in evenness (Fig. S3A), not richness (Fig.

105 S3B), suggesting an expansion of certain bacterial taxa. Indeed, we observed rapid but non-monotonic

106 changes in the relative abundances of several dominant bacterial genera, such as Bacteroides and

107 Muribaculaceae (Fig. 2C). Notably, the long-term recovery of microbiota diversity is only partial for

108 Beijing and Hunan mice (i.e. lower gut microbiota diversity at day 31 compared to day 0 ). By metagenomic

109 sequencing, we observed temporal changes in the functional capacity of gut microbiome. Specificially, the

110 initial (day 0), short-term (day 5) and long-term (day 31) microbiomes have distinct gene family profiles

111 (Fig. S4A) and the relative abundance of genes encoding enzymes for inulin utilization

112 (inulinases/fructanases) significantly increased after intervention (Fig. S4B). Collectively, our longitudinal

113 profilings are consistent with previous observations that dietary fibers have profound impacts on the

114 ecology and function of gut microbiota [36, 37]. In addition, we found the tendency of gut microbiota to

115 stabilize under sustained stimulation of inulin (Fig. 2D). The microbiota compositions at the end of inulin

116 intervention (all mice sacrificed on day 31) were clearly distinct from their baselines, indicating new

117 equilibria sustained by inulin intake.

118 The changes in the gut microbiota were accompanied by changes in the levels of three major SCFAs

119 (acetate, propionate and butyrate) and valerate (Fig. 2E, Fig. S5). Since SCFAs are metabolites produced

120 by colonic bacterial fermentation of inulin, we expect a similar phase-dependent dynamics of fecal SCFAs

121 concentration. Indeed, both total (acetate, propionate, butyrate, iso-butyrate, valerate, iso-valerate) and the

122 three major SCFAs show two temproal phases: their levels peaked in short-term before gradually decreasing

123 until steady states, with an exception of Shanghai mice whose propionate production was notably delayed

124 and compromised. The mean peak-to-baseline concentration ratios of total SCFAs were 3.3, 3.9, 4.5 and

1254.2 for Beijing, Guangdong, Hunan and Shanghai mice respectively. The long-term decline in SCFAs was

126 not a result of reduced diet intake, as the intake rate remained unchanged over time (Fig. S2). Despite

127 reduced SCFAs in the second phase, the mean concentrations of total SCFAs at day 31 were still 2.0-3.5

128 fold of its baseline levels.

129 We have shown above that Shanghai mice had a delayed increase in bacterial absolute abundance

130 (Fig. 2A) and produced low levels of propionate (Fig. 2E) in response to inulin. The distinct behavior of

131 Shanghai mice indicated that the responses of bacterial absolute abundance and SCFA production may

132 depend on the baseline microbiota. To formally test this, we separately tested for the statistical significance

133 of two orthogonal properties_- "baseline dependence" and "responsiveness" - based on the time series data 
134 of intervention group and control group (see Methods). Time series data of both groups were projected

135 onto a 2-dimensional space by sequential non-negative matrix factorization [38] to capture representative

136 temporal trends (Fig. 3A, Fig. S6). With this coarse-grained data representation, we then obtained two $P$ -

137 values by comparing the differential responses between the intervention and control group

138 ("responsiveness", $P r$ ) as well as those between the four vendors in the intervention group ("baseline

139 dependence", $\mathrm{Pb}$ ) using Permutational Multivariate Analaysis of Variance test. This approach confirmed

140 that the dynamical responses of bacterial load (Fig. 3B), propionate and butyrate (Fig. 3C) were nontrivial

141 and baseline-dependent (both $\mathrm{Pr}$ and $\mathrm{Pb}<0.05$ after multitest correction).

\section{Primary degraders of inulin respond fast and drive community dynamics}

144 We used the generalized Lotka-Volterra (gLV) model to infer key ecological drivers of the mouse gut 145 microbiota in response to dietary fiber intervention (Fig. 4A, see Methods). The gLV model assumes that 146 degradation and subsequent utilization of dietary fibers boost bacterial growth rate (the amount of increment

147 is parameterized by $\epsilon$ ). One long-standing challenge in gLV inference is overfitting of parameters when the 148 time series is too short and the number of samples at all timepoints is far less than that of gLV parameters.

149 To address the challenge, we estimated the uncertainty associated with model parameters by formulating 150 the gLV-based inference in a Bayesian framework which outputs posterior distributions of estimated 151 parameters [39], rather than point estimates in penalized regressions [40]. In our gLV-based probabilistic 152 framework, any bacteria taxa with a significantly positive distribution of "fiber response" $\epsilon$ is considered a 153 putative "primary degrader" of inulin. We identified five such bacterial taxa grouped at varying taxonomic 154 levels, including Muribaculaceae (family), Faecalibaculum (genus), Parasutterella (genus), and 155 Bacteroides (genus) and Bacteroides acidifaciens (species) (Fig. 4B), see Methods). The inference of 156 primary degraders in the gLV-based framework was robust to the criteria used for clustering $16 \mathrm{~S}$ amplicons, 157 either at the lowest classified taxonomic level (Fig. 4B) or at the OTU (Operational Taxonomic Unit, 97\% 158 sequence similarity) level (Fig. S7).

For four of the five putative inulin degraders (except Parasutterella), we found genetic evidence 160 and/or in vitro experiments from literature to support their functional roles in inulin degradation (Table S1).

161 For example, members from Bacteroides and Muribaculaceae contain PULs with a susC/susD homologous 162 gene pair that facilitates sensing and import of inulin [41, 42]. Putative inulin PULs were also detected 163 in the metagenome-assembled genomes of B. acidifaciens and Muribaculaceae (Table S2). Furthremore, 164 we analyzed the data from an independent study [43], which profiled the murine gut microbiota 165 composition after inulin intervention for two weeks. Analysis of this independent experiment found similar 166 dynamics in gut microbiota diversity and composition (Fig. S8A,B). Although bacterial absolute abudance 
was not available in this data set, we applied the Bayesian version of gLV-based inference to the relative abundance profiles and again identified B. acidifaciens as a primary degrader of inulin (Fig. S8C).

Alternatively, we used the statistical test of responsiveness (Fig. 3A; $\operatorname{Pr}<0.05$ after multitest correction) to identify all bacterial taxa that exihibited differential responses between inulin and cellulose groups, regardless of the ecological mechanism. We found a total of 37 bacterial taxa with significant

172 dynamical responses (Table S3), including the five putative primary degraders as inferred by the gLV 173 model. Among the remaining 32 "generic responders", Akkermancia muciniphila and Bacteroides 174 uniformis are the most abundant taxa whose relative abundances significantly increased after day 5 (Fig. 175 S9, Fig. S10). Unlike the gLV-based approach, the statistical analysis on time series data did not control 176 for indirect effects on bacterial growth via ecological interactions, so the two "generic responders" may 177 indirectly benefit from the primary degraders. Indeed, inference of the gLV model did not identify $A$. 178 muciniphila as a "primary degrader", but suggested that its growth was facilitated by B. acidifaciens (Fig. 4C). This is also consistent with previous observations that A. muciniphila cannot grow on inulin but can be significantly promoted by shorter chain fructo-oligosaccharides [44].

The notion of eco-group (or guild), i.e., a set of bacterial taxa that perform similar functions, is very useful to understand microbial ecology $[45,46]$. We divided the entire gut microbiota into three ecogroups: (1) 5 primary degraders of inulin; (2) 32 generic responders to inulin intervention; (3) nonresponders. The group-level dynamics showed that the primary degraders clearly dominated the microbiota response (Fig. 4D). The short-term rise in the absolute abundance of a few bacterial taxa corresponds to the initial decline of the gut microbiota evenness soon after the intervention (Fig. 2B). More interestingly, the baseline-dependent responses can be causally linked to the initial composition of key bacterial taxa (Fig. 4D,E). For example, the abundances of A. municiphila and B. uniformis increased dramatically in Hunan mice (Fig. S10), which contained the highest abundance of these two species in the baseline (dark yellow box frames in Fig. 4E). Similarly, the extremely low baseline abundances of B. acidifaciens and Muribaculaceae in Shanghai mice (violet box frames in Fig. 4E) may explain the sluggish responses in bacterial absolute abundance and SCFA productions (Fig. S5, S10).

Our gLV-based inference suggested strong competition among primary degraders, where Muribaculaceae inhibited the growth of B. acidifaciens and Facaelibaculum (Fig. 4C). Indeed, B.

195 acidifaciens and Facelibaculum showed transient dynamics with a quick rise and drop in their absolute 196 abundances, while the abundance of Muribaculaceae increased steadily and remained high during the entire 197 period of observations (Fig. 4F, S10). Our results are consistent with previous studies by Patnode et al. [47] 198 that identified competitive inhibition as the ecological mechanism for consistent dominance of Bacteroides 199 cellulosilyticus over Bacteroids vulgatus, even though both species contain fiber-processing polysaccharide 
utilization loci (PULs). Taken together, we demonstrate that primary degraders and their competitions are key drivers of the baseline-dependent ecological dynamics of microbiota response to dietary fibers.

\section{Baseline-dependent SCFA production and its association with gut microbiota composition}

The dynamics of SCFAs during inulin intervention varied substantially across different baselines (Fig. S5).

Shanghai mice produced the lowest level of propionate (Fig. S5); these mice also showed the lowest response in bacterial load (Fig. 2A, Fig.4D), due to very low abundance of some primary degraders and generic responders of inulin in the baseline microbiota (Fig. 4E). We hypothesized that these key taxa may directly contribute to propionate production and found that the baseline abundances of $B$. acidifaciens, Muribaculaceae, A. municiphila, B. uniformis were positively correlated the propionate concentration (Fig. 5A, left panel). Indeed, Muribaculaceae, A. municiphila and B. uniformis have been previously found to produce propionate in vitro and/or in vivo (Table S1). As a result and consistent with a previous study [48], there was a strong positive association between bacterial load and propionate concentration (Fig. 5A, right panel; $\mathrm{P}<0.001$ ), as the two are both baseline-dependent. In contrast, the association between bacterial load and other SCFAs was not significant (Fig. S11).

Given that the gut microbiota is strongly associated with the fecal levels of fiber fermentation products, we asked whether we could quantitatively predict SCFA concentrations from the microbiota composition measured at the same time. We evaluated the performance of machine learning models to predict the fecal SCFA concentrations using absolute abundance of bacterial taxa as predictors. All mice in our experiments were split into training data and test data using different data-split approach (Fig. 5B). The "interpolation" approach generated balanced distribution of baseline microbiota composition between the training and test data (Fig. S12A), by randomly selecting a single mouse from each vendor as test data and using the other mice for training. In contrast, the "extrapolation" approach produced highly unbalanced microbiota distribution between the training and test data (Fig. S12B), by randomly selecting all mice from a vendor as test data and using mice of the other vendors for training. Although the Random Forest regression model fitted the training data reasonably well $\left(\mathrm{R}^{2} \geqslant 0.66\right.$ regardless of SCFAs and data-split approaches), the predictions generalized poorly to the test data: $\mathrm{R}^{2}$ of SCFAs ranged from 0.1 to 0.45 for "interpolation" but dropped below 0 for "extrapolation" (Fig. 5C). We further showed that the low predictability in extrapolation cannot be substantially improved by using alternative predictors (Fig. S13A), models (Fig. S13B) or adding weights to training samples (Fig. S13C). Given the current sample size, we found that Random Forest regression models based on gut microbiota composition had low or no predictive power for fecal SCFA concentration, if the gut microbiota of interest was significantly different from the baselines covered in training data. This agrees with previous studies in humans finding[49] that the 
233 substantial inter-individual variation of gut microbiome could impede the predictive power of machine

234 learning models.

\section{Response to resistant starch validates model developed from response to innulin}

To study whether our ecological framework can be generalized to study the dynamical responses of gut microbiota to other dietary fibers, we administered resistant starch from maize to mice from the same four vendors following the same experimental procedure (see Methods, Fig. 1B). Compared to inulin, resistant starch stimulated milder changes in the bacterial load (Fig. 6A), gut microbiota composition (Fig. 6B), and SCFAs production (Fig. 6C). Under resistant starch intervention, we identified Faecalibaculum and Muribaculaceae as "primary degraders" (Fig. 6D, see Table S1 for genetic evidence from literature) and 25 additional bacterial taxa as "generic responders" (Table $\mathbf{S 3}$ ). The dynamics of primary degraders were qualitatively similar between inulin and resistant starch interventions (Fig. 6D): Muribaculaceae increased rapidly and reached a plateau (except for Shanghai mice), while Faecalibaculum declined sharply after the initial burst. The gLV-based inference suggested that the observed dynamics was driven by mutual inhibition between the two primary degraders (Fig. 6E).

We found that bacterial load (Fig. S14A) and the three major SCFAs (Fig. S14B) exihibited baseline-dependent dynamical responses to resistant starch intervention. For example, the weak response in bacterial load and SCFA production of Shanghai mice (Fig. 6A) could be explained by the low abundance of Muribaculaceae in the baseline community (Fig. 6F, highlighted in red box frame). In addition, there was substantial growth of generic responders in Hunan mice (Fig. 6G), although the dominant bacterial taxa in this eco-group were different from the taxa identified in inulin intervention (Table S3).

Finally, we found that the baseline abundance of Muribaculaceae was separately correlated with bacterial absolute abundance and prioprionate level (Fig. 6H, left panel), supporting the hypothesis that Muribaculaceae may serve as both a primary degrader and a propionate producer. As a result, there was a weak but statistically significant positive association between bacterial load and propionate concentration (Fig. 6H, right panel; $\mathrm{P}=0.002$ ). Similar to our findings from the inulin intervention group, Random Forest models based on gut microbiota compostion had low or no predictive power for SCFA concentration in the resistant starch intervention group (Fig. 6I). Collectively, our major findings were qualitatively consistent between inulin and resistant starch interventions, suggesting that the dynamical responses of gut microbiota to fiber-based perturbation follow universal ecological principles.

\section{Discussion}

265 Our study characterizes the ecosystem response of murine gut microbiota to fibers, and emphasizes that ecological interactions play a key role in the personalized impact of dietary changes. gLV-based ecological 
267 inference from gut microbiome time series data has yielded mechanistic insights into the stability of 268 probiotic community under dietary perturbation [49], colonization resistance of pathogenic Clostridioides 269 difficile [51], and community assembly dynamics within preterm infant gut [24]. By integrating gLV model 270 with Bayesian regression, we inferred a competitive network of fiber degraders as key bacteria that mediate 271 the response of murine gut microbiota to inulin and resistant starch intervention. Besides evidences 272 supporting the fiber-degrading function of putative degraders, our study confirms findings in the literature 273 and advances the understanding of the effects of dietary fibers on the gut microbiome at the system level. 274 First, the small number of fiber degraders (five for inulin and two for resistant starch) suggested that fiber275 induced bacterial shifts are very selective and occur to a restricted number of taxa. Second, the absolute 276 abundance of many fiber-degrading bacteria such as taxa related to the genus Bifidobacterium, failed to 277 expand in the mouse gut on both fibers (Fig. S15), indicating that fiber-induced bacterial enrichment cannot 278 be simply predicted from their in vitro growth. Third, we reasoned that the personalized fiber-induced 279 response of gut microbiota were largely determined by the baseline abundance of fiber degraders and the 280 ecological interactions among these degraders. In our study, the inter-individual variability of fiber-induced 281 shift in propionate production was associated with the baseline abundance of a key fiber degrader 282 Muribaculaceae. This is consistent with previous findings that the abundance of a key starch-degrader prior 283 to resistant starch intervention was indicative of whether an individual would have higher fecal butyrate 284 response [16]. The ecological interactions among fiber degraders, on the other hand, drives the cascading 285 alterations during the intervention. Our results revealed that fiber-induced dynamics of murine gut 286 microbiota were largely driven by competitions and Muribaculaceae outcompeted other degraders in 287 consuming both inulin and resistant starch. Since the family Muribaculaceae was specific to the mouse gut 288 [52], it might have been adapted to the murine gut with higher fitness.

289 Understanding the microbial responses and its association with the baseline microbiota 290 composition rightly is a critical step in individualized dietary fiber intervention. To date, most of the current 291 studies are based on cross-sectional study design and described the pre-to-post changes in 292 abundance/concentration as microbial responses to dietary intervention $[13,15]$. However, it should be 293 noted that gut microbiome is a highly dynamic ecosystem, and its response to dietary fibers could have 294 temporal characteristics [37]. Consequently, the significance of microbial responses may vary depending 295 on the study endpoint used to calculate pre-to-post changes. In our experiments, the changes in propionate 296 concentration from their baseline levels differ significantly among the four vendors at day 5 but not at day 29731 (Fig. S16). Furthermore, due to the lack of control group data to assess the intervention effects, pre-to298 post changes that are supposedly to capture fiber-indcued effects may be entirely attributable to random 299 temporal variations within each individual [53]. We speculate that these two caveats are the main cause of 300 "reproducibility crisis" [54] among microbiome researches. In constrast, our analysis avoids the above two 
caveats by incorporating longtitudinal data and a control group. Additionally, the use of dimensionality reduction in our approach further benefits data visualization of inter-vendor variations in gut microbiota composition (Fig. 3).

Diet-induced changes in SCFAs are often transient and vanish shortly after cessation of dietary intervention [9, 55-57]. Our study is consistent with this result, by showing that SCFA concentrations cannot be maintained at its peak and drop by $35 \%-40 \%$ even under continuous inulin intake until four weeks. The transient responses in SCFAs were also observed in colorectal cancer patients [58] and type 2 diabetes mellitus patients [6]; however, it is unknown whether the reduced SCFAs in these human subjects are resulted from lower dietary fiber intake. Despite the drop, our data demonstrates that a continuous intervention that lasts for 31 days is sufficient to elevate and stabilize the SCFAs concentration, but it is not clear yet whether the elevated level persists after the intervention discontinues. The in vivo SCFAs dynamics is jointly determined by multiple metabolic processes, where the two major ones are microbial production and host absorption. In healthy individuals, 90\%-95\% SCFAs produced in the colonic lumen are absorbed by the gut mucosa [59]. While many studies used fecal SCFAs concentrations as proxy of their luminal levels, neither of both represents the rate of production or absorption so the declined phase of SCFAs in our study may be explained by reduced production rate, increased absorption rate or both. Due to the difficulty of measuring fluxes in vivo, mathematical models that take both processes into accounts show great premise in the estimation of their flux rates from SCFAs concentrations [60].

Characterizing the dynamics of gut microbiota and their inter-individual variability with multiomics data is an important priority for microbiome research to further understanding of diet-induced responses [61]. Such studies hold great promise to improve human health and treat gut microbiomeassociated disease via microbiome engineering. A key question in microbiome engineering with prebiotics is whether and to what extent can we enrich the gut levels of beneficial bacteria using prebiotic compounds.

324 Microbiome engineering, as with other engineering disciplines, requires computational tools to aid the 325 design process. Predicting bacterial responses to interventions in the human gut is nontrivial: previous 326 studies have repeatedly shown that bacteria able to consume a fiber supplement in vitro may not be 327 selectively enriched in vivo, suggesting that dietary response of a gut bacterial taxa depends on the 328 ecological context [62]. By inventing a new application of gLV with uncertainty assessment to infer primary 329 fiber degraders and the associated interaction network, we provide a generalizable computational approach 330 to study the ecological dynamics of the gut microbiome under dietary interventions. We foresee that 331 applications of ecological modeling in human cohorts with dense longitudinal sampling will provide 332 important insights for predictable dietary responses and personalized nutrition [63]. 
A

B
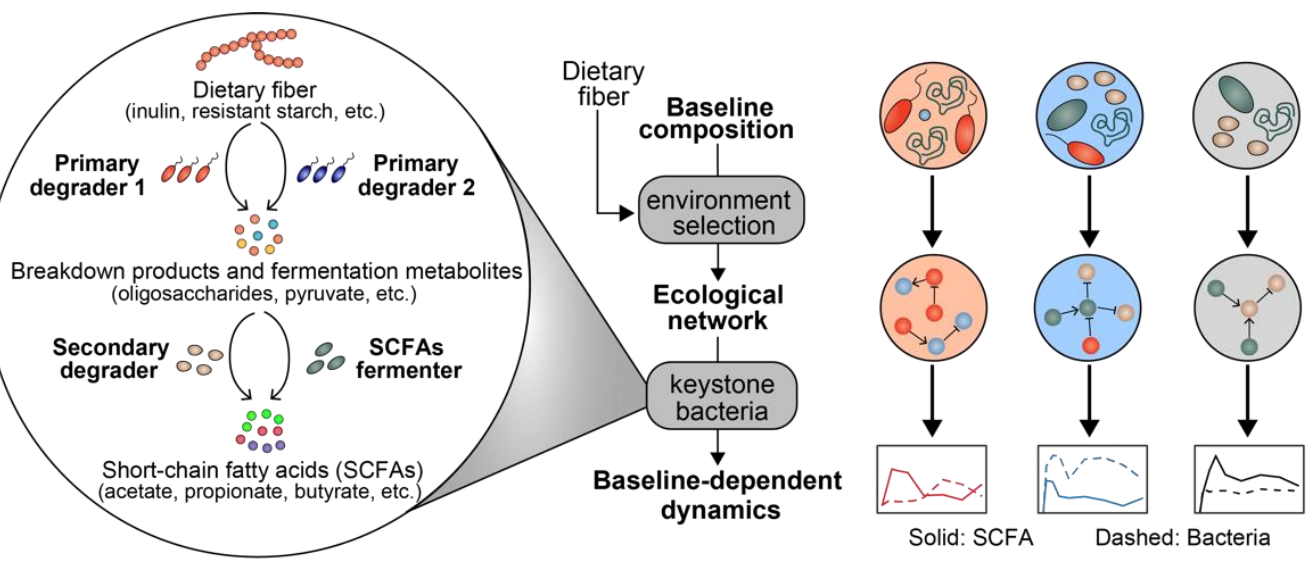

Solid: SCFA

Dashed: Bacteria

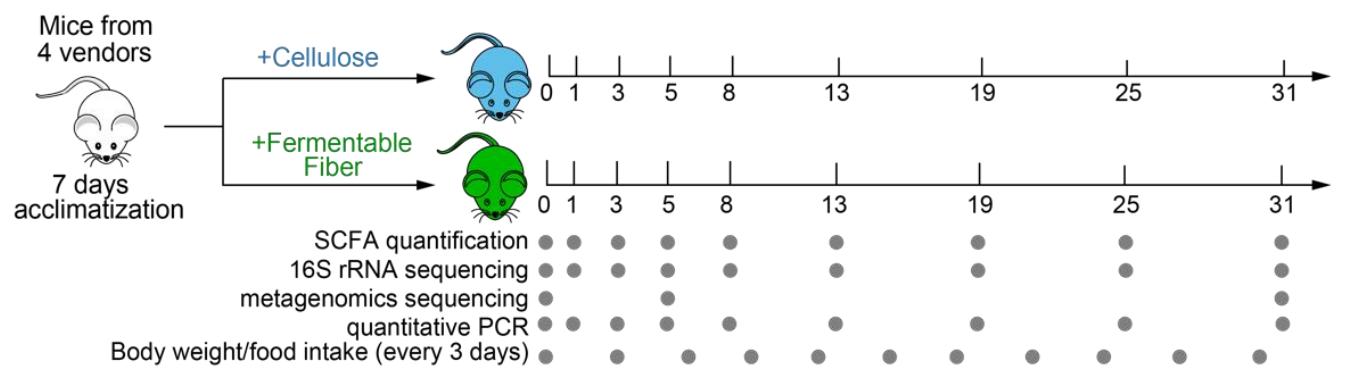

C

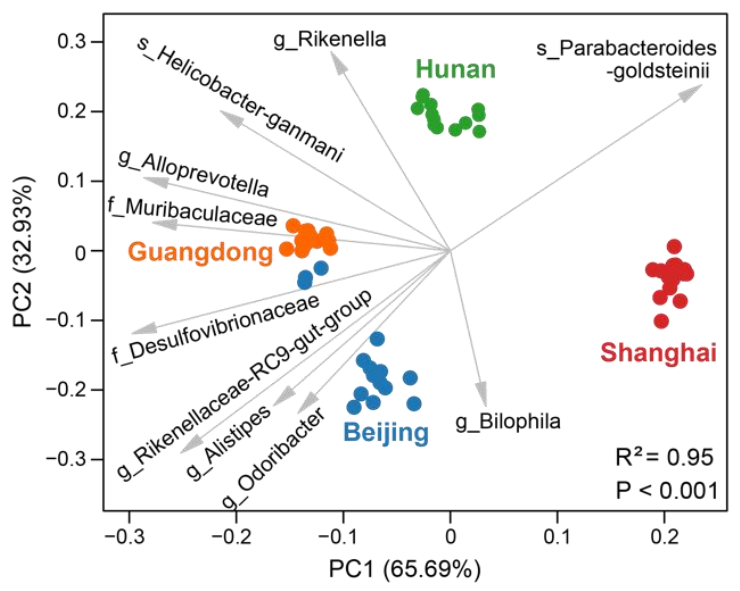

D

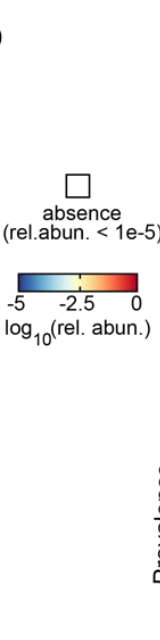

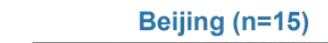

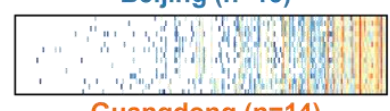

Guangdong $(n=14)$

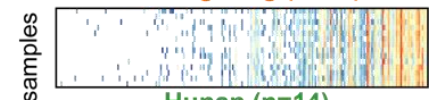

Hunan $(n=14)$
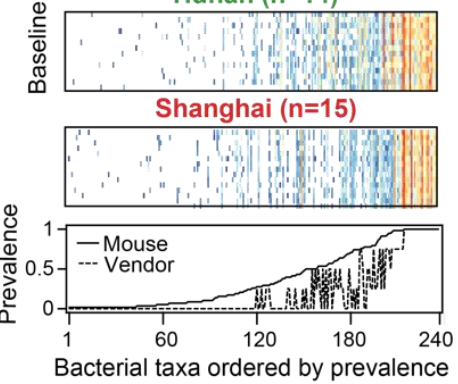

Figure 1. Longitudinal profiling of murine gut microbiota and metabolites to study the baselindependent dynamics in response to dietary fiber intervention. A. An ecological perspective of baselinedependent dynamical responses of gut microbiota and SCFAs. Administration of dietary fibers alters ecological substrate niche in the gut and selects for a unique ecological network for each baseline microbiota type. Within the network, a few gut bacteria playing key metabolic roles as primary/secondary degraders and SCFAs fermenters drives heterogenous responses of bacteria and SCFAs via diverse ecological interactions (e.g., resource compeitition and cross-feeding). B. Experimental design. All mice from the four vendors were continuously fed with either dietary fiber (inulin or resistant starch)- or cellulosesupplemented diets for four weeks. Gray dots indicate the days on which data were collected from fecal 
345 samples. C. Baseline microbiota composition shown in robust PCoA (principal coordinate analysis) biplot.

346 Isogenic age- and gender-matced mice were purchased from four different vendors (Beijing, Guangdong,

347 Hunan, Shanghai). Gray arrows represent the dominant bacterial taxa in these samples. Adonis analysis

348 was performed to test for differences in baseline gut microbiota composition across the four vendors

349 ( $\mathrm{P}<0.001)$. D. Top four panels: presence (white blocks indicate absence) and abundance (colored blocks)

350 of bacterial taxa in the baseline samples. Bottom panel: the prevalence score of a taxon across mice (defined

351 as the fraction of all mice that contains this taxon) or vendors (defined as the fraction of vendors whose

352 mice all contain this taxon). 

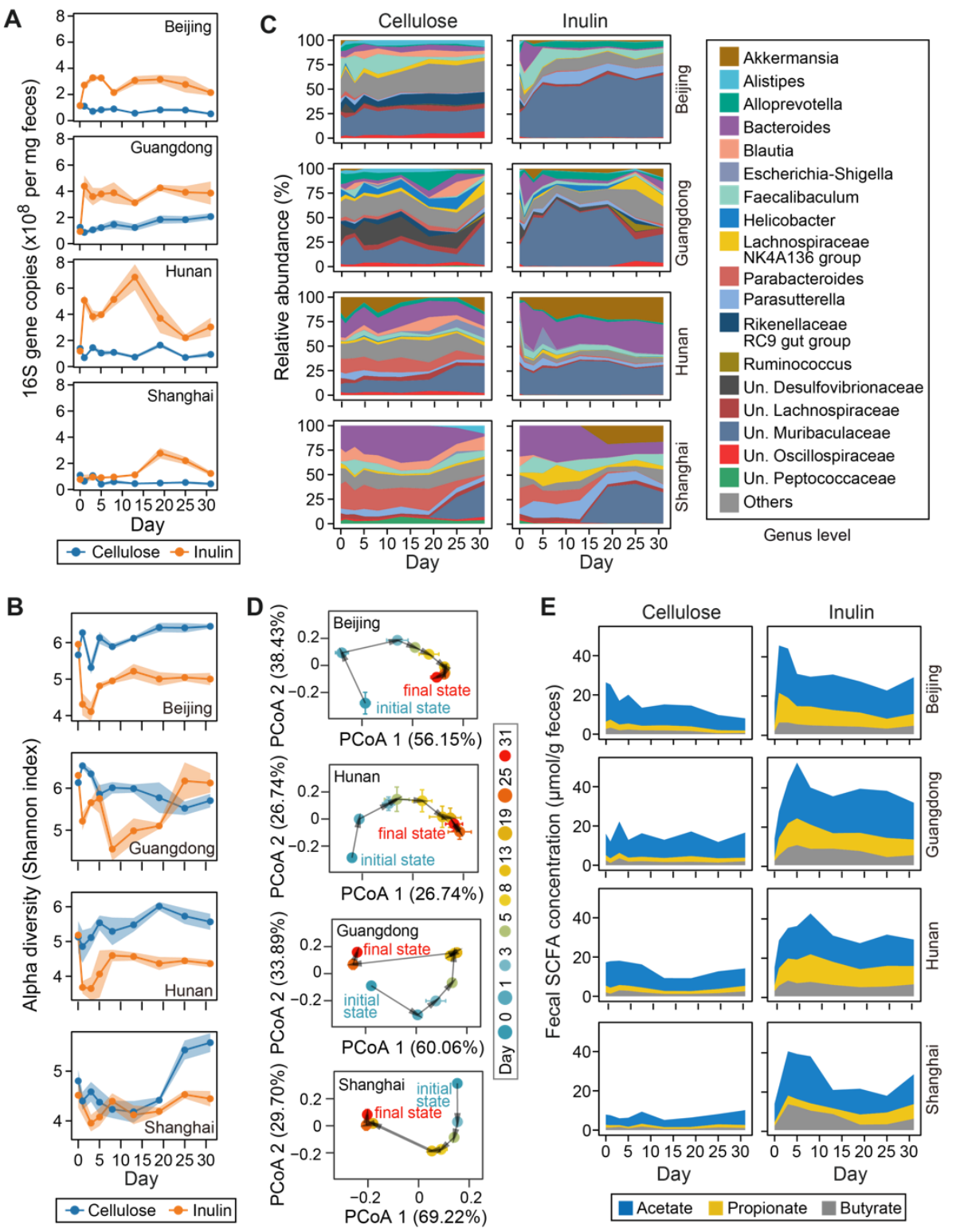

Figure 2. Inulin-induced temporal shifts in murine gut microbiome and short-chain fatty acids (SCFAs) metabolism. A. Bacterial load. B. Alpha diversity of gut microbiota composition. C. Relative abundance of bacterial genera shown in stacked band plot. Un.: unclassified. D. Shifts in gut microbiota composition represented by PCoA (principal coordinate analysis) plot. Initial and final states represent the microbiota compositions at day 0 and day 31 respectively. E. Fecal concentration of acetate, proprionate and butyrate. Beijing, Guangdong, Hunan, Shanghai are four different mice vendors. Lines (panels A,B), dots (panels A,B,D), and stacked bands (panels C, E) represent the mean values over mouse replicates from the same vendor ( $n=4$ for Hunan and Guangdong, $n=5$ for Beijing and Shanghai). Shading areas (panels A,B) and error bars (panel D) represent standard error of the mean. 
A

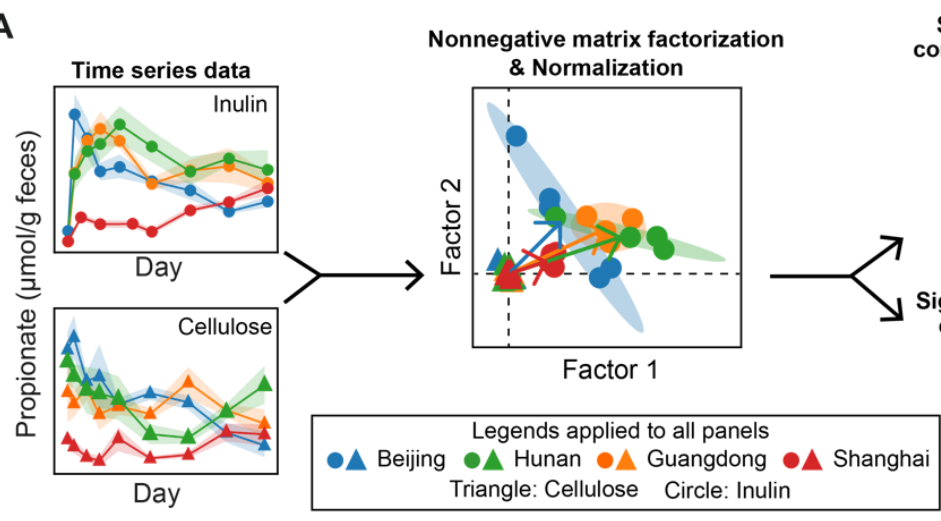

Sig. response $(\mathrm{Pr}<0.05) ?$

compare inulin and cellulose

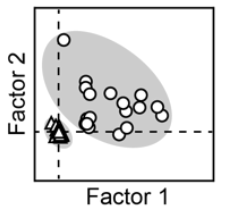

Sig. baseline dep. $(\mathrm{Pb}<0.05)$ ? compare across vendors

Figure 3. Quantifying the statistical significance of baseline-dependent dynamical response. A. A schematic diagram of our statistical framework to test for the significance of baseline-dependent response. The framework involves three steps: (1) projecting all time series from both intervention and control groups onto the same 2-dimensional space, (2) normalizing all data points by recentering the mean of control group to the origin, and (3) performing two separate statistical tests using the projected data to quantify the significance of "responsiveness" $(\mathrm{Pr})$ and "baseline-dependence" $(\mathrm{Pb})$ using Permutational Multivariate

372 Analaysis of Variance test. Abbreviations: Significant (Sig.); dependence (dep.). For each baseline (Beijing,

373 Guangdong, Hunan, Shanghai), an arrow was drawn from the eclipse center of the baseline under cellulose

374 intervention (standardized to the origin) to that under the inulin intervention. B,C. Reduced 2-dimensional

375 representation of the inulin-induced responses in bacterial load (B) and three major SCFAs (C). In all panels, 376 each symbol represents a mouse (triangles: cellulose group, circles: inulin group) and all mice data from 377 the same vendor under the same intervention (inulin or cellulose) was used to fit an eclipse (ellipse's radius 378 was determined by 2 standard deviations). 
A

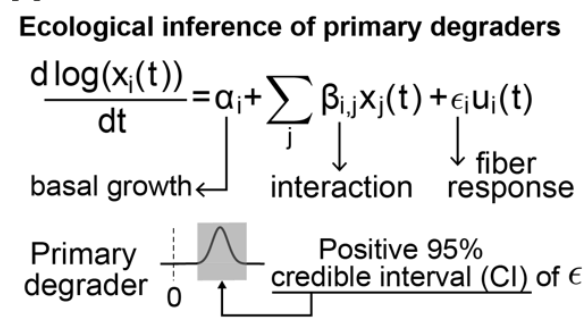

D

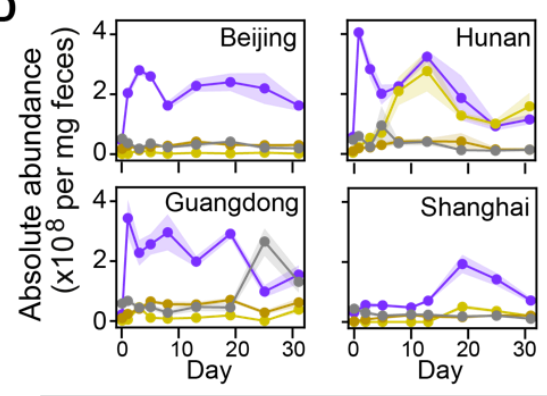

- Primary degraders $\rightarrow$ Non-responders
- Generic responders (A. muc and B. uni)
- Other generic responders
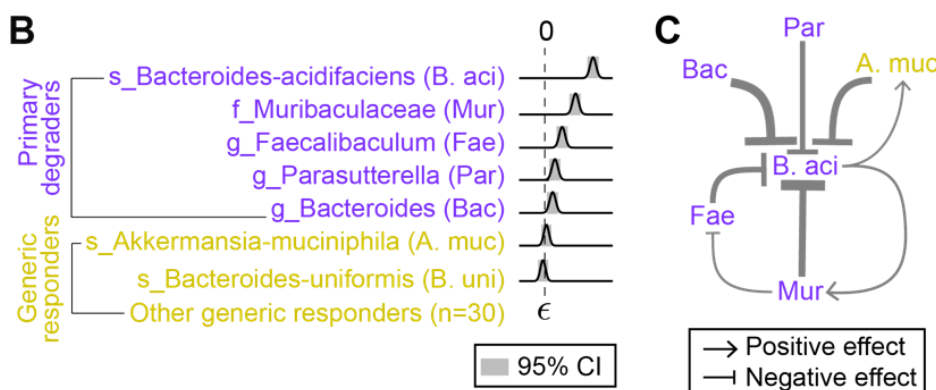

E

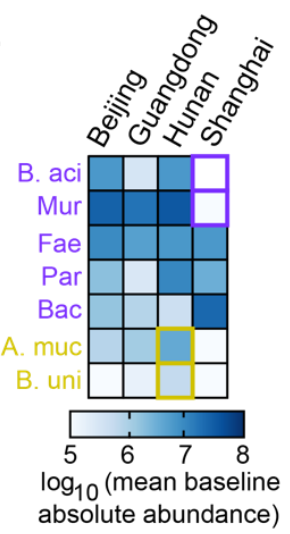

$\mathbf{F}$

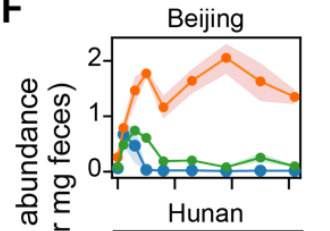

$\rightarrow$ Positive effect $\rightarrow$ Negative effect
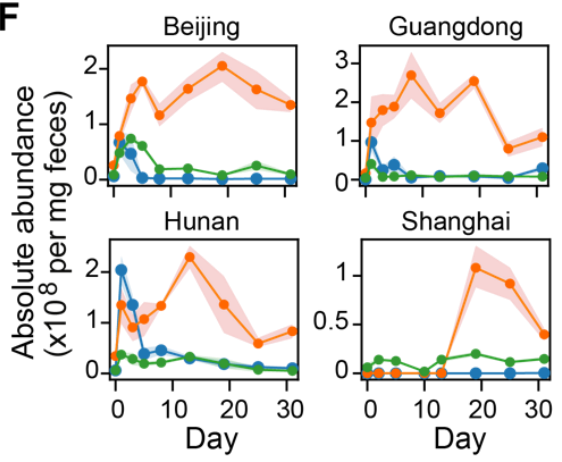

$\rightarrow$ B. aci $\rightarrow$ Mur $\bullet-$ Fae

Figure 4. Inulin responders shape the dynamical response of murine gut microbiota. A. Generalized

Lotka-Volterra (gLV) model combined with Bayesian statistics to infer inulin degraders and pairwise interactions. The gLV model summarizes the underlying ecology by three terms that additively determine bacterial growth rates: the basal growth rates $(\alpha)$, the influences from other bacteria $(\beta)$, and the impacts of dietary fiber $(\epsilon)$. A primary degrader is determined when $95 \%$ credible interval of the posterior distribution of $\epsilon$ is positive. B. Posterior distribution of $\epsilon$ for five primary degraders (violet) and two generic responders (dark yellow). Generic responders are those bacteria showing statistical significance of inulin-induced response (i.e., responders) but not inferred as primary degraders. Bacterial taxa are ranked according to their posterior mean of $\epsilon$. C. Core ecological interaction network composed of six bacterial taxa shown in the panel B (see all significant interactions in Table S4). Point and blunt arrows represent positive and negative interactions respectively. The arrow thickness is proportional to the posterior mean of the corresponding interaction coefficient. D. Ecological group dynamics of primary inulin degraders, generic responders (presented with two subgroups) and non-responders. E. Mean absolute baseline abundance of the seven bacterial taxa shown in the panel B. F. Temporal changes in the absolute abundance of the top three inulin degraders. In panels D, F, lines and dots represent the mean values across mice from the same vendor ( $\mathrm{n}=4$ for Hunan and Guangdong, $\mathrm{n}=5$ for Beijing and Shanghai). Shading areas represent the standard error of the mean. 
A
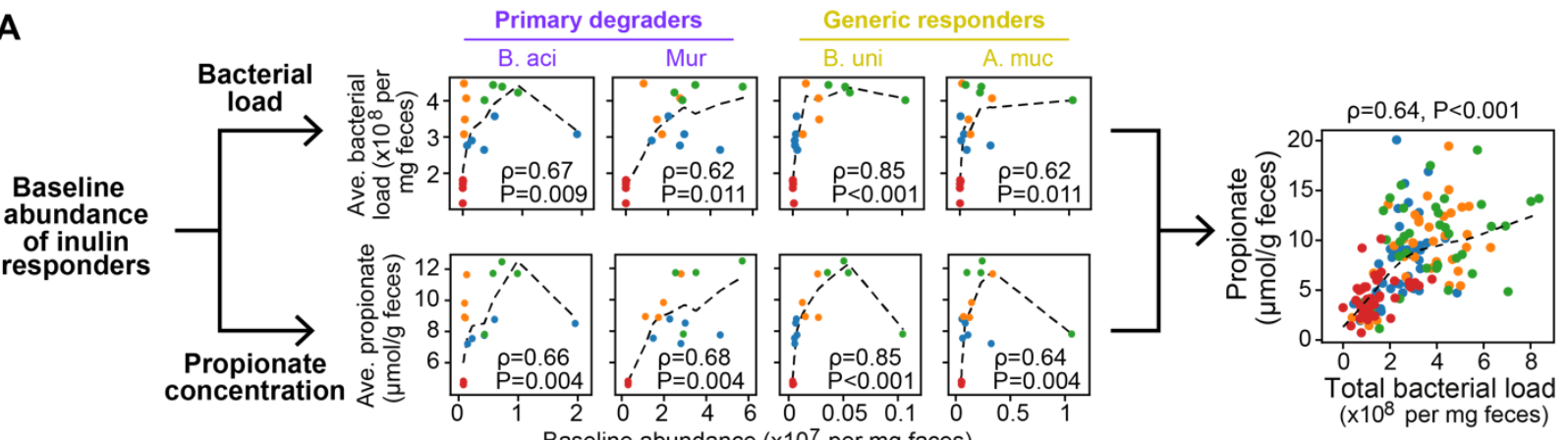

B

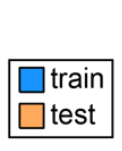

Random Forest model

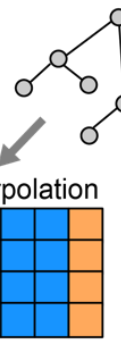

Individual groups

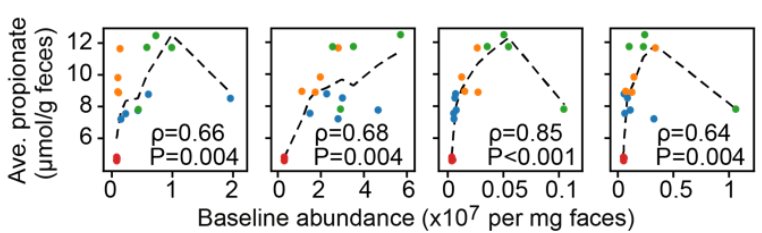

$\bullet$ Beijing $\bullet$ Hunan $\bullet$ Guangdong $\bullet$ Shanghai
C

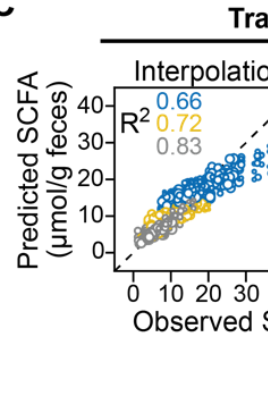

Training data

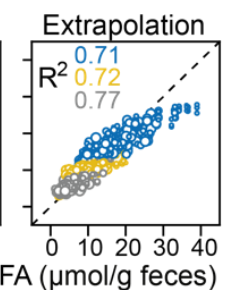

(

Day: $\bullet 1,6$ Acetate $\bigcirc$ Propionate $\bigcirc$ Butyrate

Test data

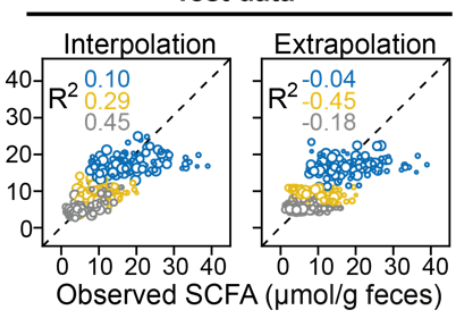

Individual groups

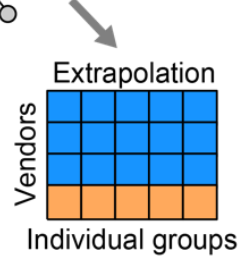

Figure 5. Quantitative relationship between SCFAs and murine gut microbiota composition. A.

401 Correlation between bacterial load and propionate concentration (right big panel). We proposed that the correlation is mediated by some inulin responders which causally and simultaenoulsy affect both observations. Eight small panels to the left: Spearman's correlations of baseline abundance of four inulin responders (B. aci: s_Bacteroides-acidifaciens, Mur: f_Muribaculaceae, B. uni: s_Bacteroides-uniformis, A. muc: s_Akkermansia-muciniphila) with the mean bacterial load (top row) or propionate concentration (bottome row) averaged across the interveion period. Dashed line: Lowess (Locally Weighted Scatterplot Smoothing) regression. Spearman correlation coefficient ( $\rho)$ and adjusted P-value are indicated in each plot. B. Prediction of SCFAs concentrations from gut microbiota composition using machine learning models. Two data-split strategies for testing model performance were designed: mice in the test sets were randosmly selected on a one-per-vendor basis for "interpolation" and exclusively selected from a single vendor for “extrapolation”. Data before intervention (i.e., day 0) was not included. C. Performances of Random Forest regression models on the training and test datasets. 

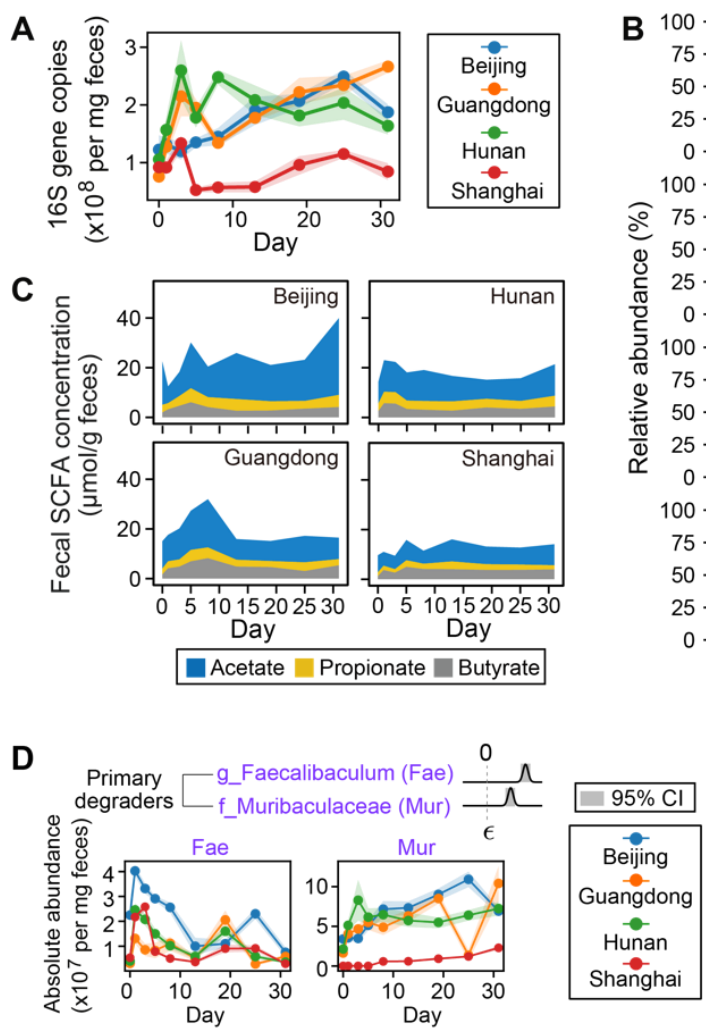

H
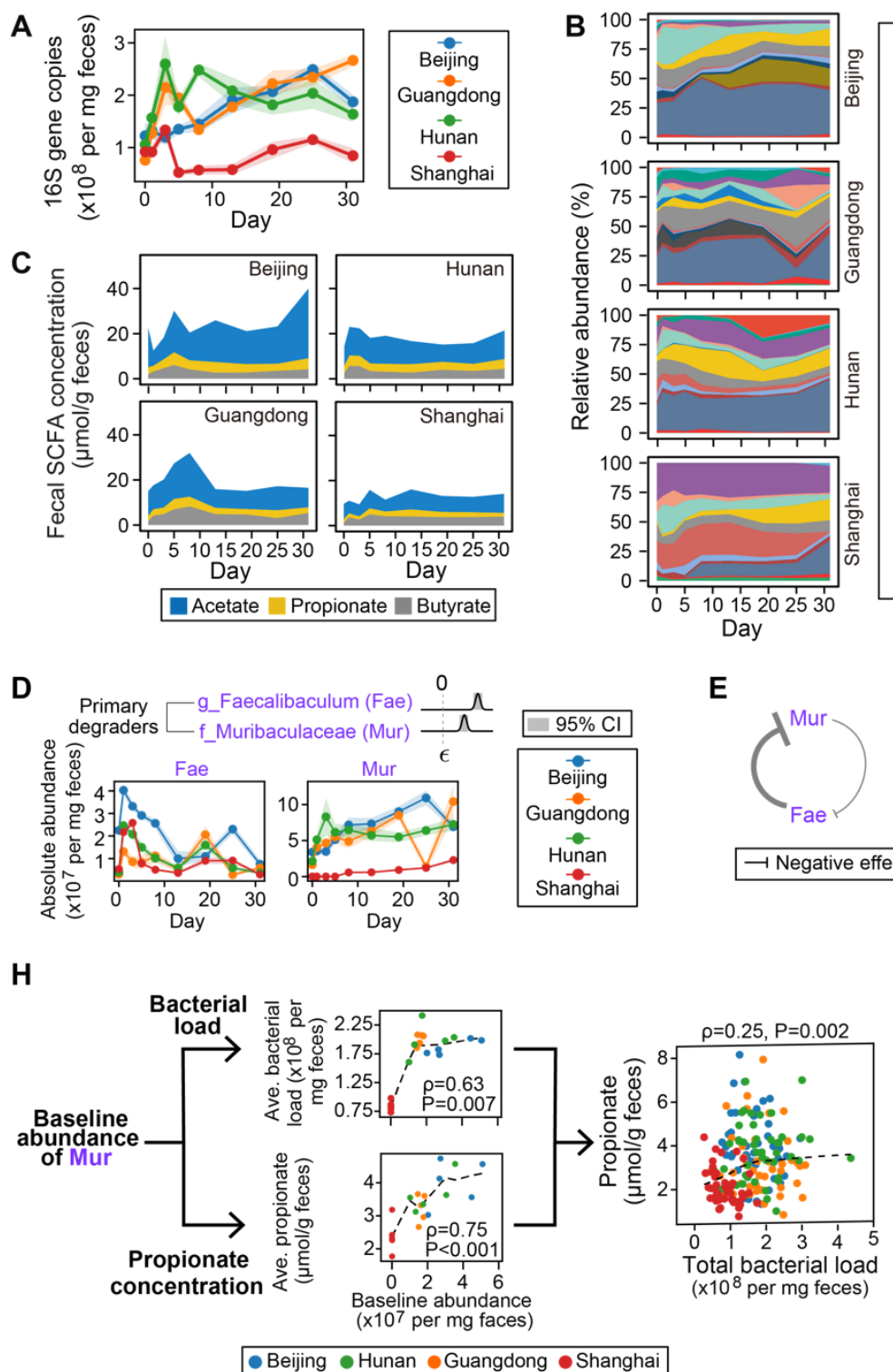

E
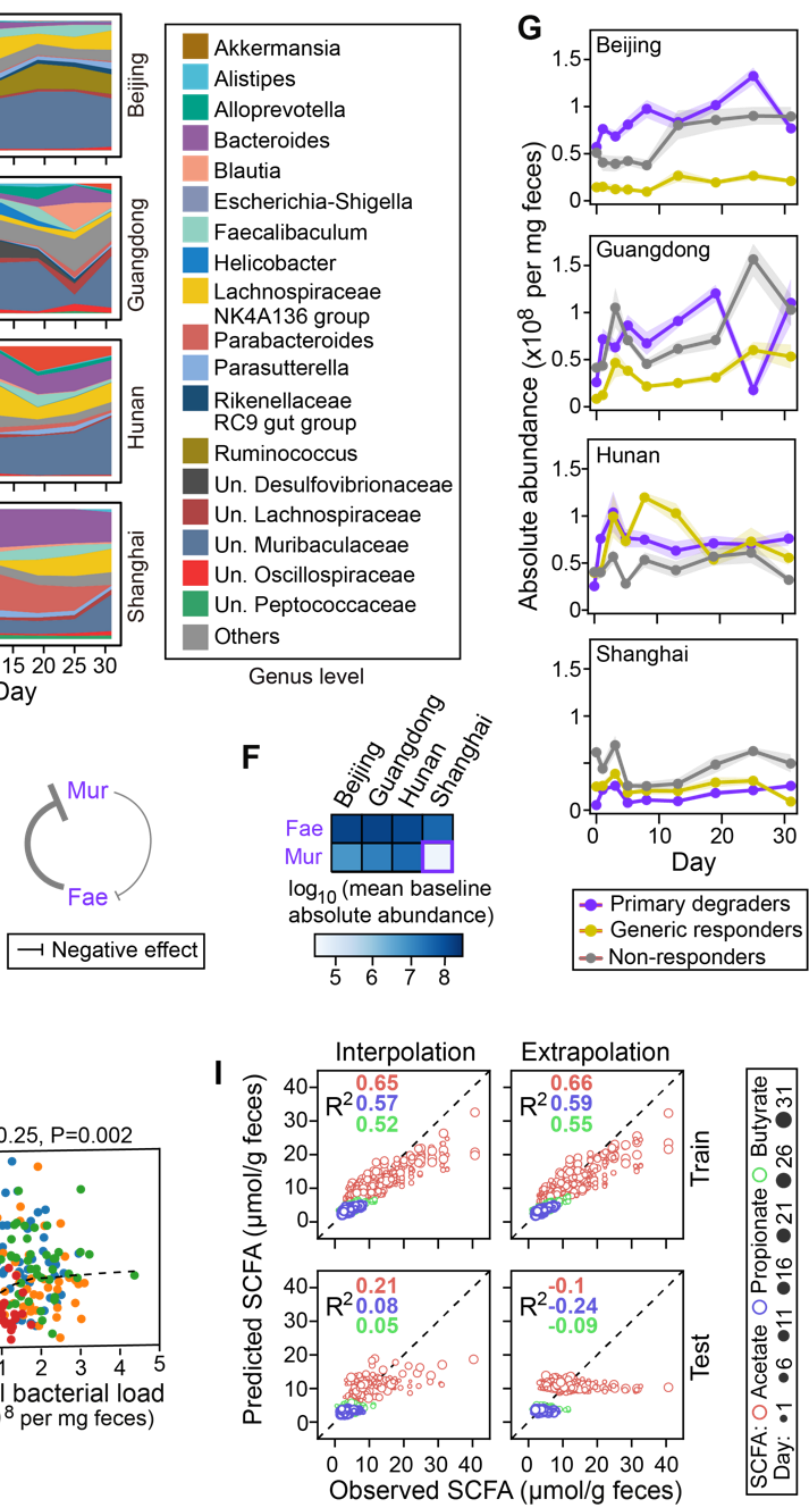

Figure 6. Resistant starch-induced dynamical response in murine gut microbiota. A-C. Dynamical responses of bacterial load (A), gut microbiota composition (B), and SCFAs concentration (C) following resistant starch intervention. D. Dynamics of two putative resistant starch degraders. $\epsilon$ represents the growth impact of resistant starch and its posterior distributions are shown for each degrader. CI: credible interval. E. Ecological interactions between the two degraders (see all significant interactions in Table S4). The arrow thickness is proportional to the posterior mean of the corresponding interaction coefficient. F.

421 Mean baseline abundances of the two degraders. G. Ecological group dynamics of primary degraders and 422 generic responders of resistant starch as well as the non-responders. H. Correlations among baseline 423 abundance of Muribaculaceae, bacterial load and propionate concentration. For the two small panels on the 424 left, the time-averaged bacterial load or propionate concentration on the y-axis were calculated by dividing 425 the area under the curve of the corresponding variables by the duration of observation. Dashed lines: Lowess 
426 (Locally Weighted Scatterplot Smoothing) regression. I. Prediction of SCFAs concentration from gut 427 microbiota compsotion using a Random Forest regression model. "Interpolation" and "extrapolation” are 428 two strategies of splitting all data into the training and test sets (the same as Fig. 5B). Lines (panels A,D,G) 429 or height of stacked bands represent mean values across mice from the same vendor ( $\mathrm{n}=5$ for all vendors). 430 Shading areas (panels A,D,G) represent the standard error of the mean. 


\section{Methods}

435 Animal experiments. Specific-pathogen-free (SPF) female C57BL/6J mice were obtained at 6 weeks of 436 age from four different vendors, including Beijing (A Charles River Company, Beijing, China), Hunan 437 (Hunan Slac Jingda Laboratory Animal Company, Ltd., Changsha, China), Guangdong (Guangdong 438 Medical Laboratory Animal Center, Foshan, China)) and Shanghai (SLAC Laboratory Animal Co., Ltd., 439 Shanghai, China). Mice were maintained in 12-h light/dark cycle and allowed ad libitum access to food and 440 water throughout the experiment. After acclimatizing to the diet and housing environment for 1 week, mice 441 from each vendor were randomly separated into three groups: cellulose group $(n=5)$, resistant starch group $442(n=5)$, and inulin group $(n=5)$. Composition of all diets including the source of dietary fibers cellulose, 443 resistant starch from maize (HI-MAIZE® 260, Ingredion Inc.), and inulin (Orafti HP, BENEO-Orafti) are 444 provided in Table S5. Fecal pellets from each mouse were freshly collected over multiple time points: day 4450 (before diet change), day 1, 3, 5, 8, 13, 19, 25, and 31 (Figure 1A). Fecal samples were snap-frozen in 446 liquid nitrogen and stored at $-80^{\circ} \mathrm{C}$ until further processing. At every cage change (moving the mice to a 447 new clean cage with fresh bedding twice in one week), body weight was individually measured, and food 448 intake and fecal output of each cage mice during the previous three days per cage were measured. This 449 study was approved by the Institutional Animal Care and Use Committee of the Shenzhen Institutes of 450 Advanced Technology, Chinese Academy of Sciences.

452 Quantification of fecal SCFA concentration by GC-MS. The SCFAs of mice fecal samples were 453 analyzed by GC-MS [63]. For the sample extraction, $0.05 \mathrm{~g}$ of frozen feces were mixed with $300 \mu \mathrm{L}$ of pure 454 water containing caproic acid-6,6,6-d3 (CDN Isotopes, Quebec, Canada) as internal standard (final 455 concentration $20 \mu \mathrm{g} / \mathrm{mL}$ ). After adding $1.0 \mathrm{~mm}$ diameter zirconia/silica beads (BioSpec, Bartlesville, OK), 456 feces were homogenized for $20 \mathrm{~s}$ under $6500 \mathrm{rpm}$ for three times, then incubated at $4{ }^{\circ} \mathrm{C}$ with shaking for $45730 \mathrm{~min}$, followed by centrifugation for $30 \mathrm{~min}$ at $13,000 \times \mathrm{g}$. Following extraction with anhydrous diethyl 458 ether, the SCFA extract accurately transferred into a glass insert in a GC vial and capped tightly after added $4595 \mu \mathrm{l}$ of $\mathrm{N}$, O-bis(trimethyl-silyl)-trifluoroacetamide and vortexed for $5 \mathrm{~s}$. The mixture was kept in the GC 460 vial and incubated at room temperature $\left(22^{\circ} \mathrm{C}\right.$ ) overnight (or over $8 \mathrm{~h}$ ) before loading to GC/MS. The 461 analysis of acetic, propionic, iso-butyric, iso-valeric, valeric and butyric acids was performed by Agilent 462 8890/7000D triple quadrupole GC/MS equipped with a capillary HP-5 ms capillary column $(30 \mathrm{~m} \times 0.25$ $463 \mathrm{~mm} \times 0.25 \mu \mathrm{m}$ film thickness) (Agilent Technologies). The analytes were quantified in the selected ion 464 monitoring (SIM) mode using the target ion and confirmed by confirmative ions. The integrated areas for 465 all SCFAs were normalized with the internal standard and quantified with the standard curve constructed. 
DNA extraction and quantification of bacterial load. DNA of mice fecal samples was extracted using the QIAmp PowerFecal DNA kit (Qiagen,\#12830-50) following standard manufacturer procedures. DNA samples were resuspended in Buffer C6 and quantitated using the Qubit fluorometer (ThermoFisher Scientific). To quantitatively assess bacterial load, total bacteria density were determined using qPCR as previously described [66]. A 466-bp fragment of the bacterial 16S ribosomal DNA was amplified using the

472 forward primer 5'-TCCTACGGGAGGCAGCAGT-3' and the reverse primer 5'473 GGACTACCAGGGTATCTAATCCTGTT-3'. The absolute abundance of a bacterial taxon was estimated 474 by multiplication of its relative abundance and the total bacterial load.

16S rRNA amplicon sequencing and shotgun metagenomic sequencing. 16S rRNA gene sequencing was performed as previously described with modifications [67]. Library preparation was done using a twostep PCR method. During the first step of PCR, primers S-D-Bact-0341-b-S-17 (forward) and S-D-Bact0785-a-A-21 (reverse) were used to target and amplify the v3-4 region [68], as well as to add second-step priming sites. Dual index codes were added to each sample at the second PCR step. The PCR products were purified with Agencourt AMPure XP magnetic beads (Beckman Coulter, Brea, CA, USA) and quality controlled with TapeStation (Agilent Technologies, Santa Clara, CA, USA). The final DNA concentrations of the purified products were measured with Qubit 2.0 fluorometer (Thermo Fisher Scientific). The purified products were pooled in equal molar concentrations, and denatured following the Illumina protocol. Sequencing was performed in a single run on NovaSeq 6000 (Illumina, USA). Blank controls (no sample added, processed routinely, $n=4$ ) were included in the extraction process to control for contamination throughout processing.

Metagenomic sequencing was performed using fecal samples from the inulin diet group at day 0, 5 and 31. Extracted DNA sample was purified using silica-based columns. Metagenomics sequencing libraries were prepared with at least $2 \mu \mathrm{g}$ of total DNA using the Nextera XT DNA sample Prep Kit (Illumina, San Diego, USA) with an equimolar pool of libraries achieved independently based on Qubit 2.0 fluorometer results combined with SYBR Green quantification (Thermo Fisher Scientific, Massachusetts, USA). The indexed libraries were sequenced on NovaSeq 6000 (Illumina, USA) by Guangdong Magigene Biotechnology Co.,Ltd. (Guangzhou, China).

Bioinformatics analysis. The 16S rRNA sequencing reads were analyzed by QIIME 2 (version 2020.2) [69]. Demultiplexed paired-end reads were trimmed to remove primers and low-quality bases with q2cutadapt plugin. The trimmed sequences were denoised and joined with q2-dada2 plugin. Potential reagent contaminants were identified using decontam package based on either the frequency of the amplicon sequence variants (ASVs) in the blank control or the negative correlation with DNA concentration [70]. 
The generated feature table was filtered to remove ASVs present in only a single sample and remaining ASVs were used to construct a rooted phylogenetic tree via q2-phylogeny. Rarefaction curve analysis of the data obtained was used to estimate the completeness of microbial communities sampling and performed using the iNEXT R package [71]. Subsequently, in order to avoid sample-to-sample bias due to variable sequencing depth (different number of reads per sample), samples were rarefied to 38,980 sequences per sample. Rarefaction analysis showed that great majority of the bacteria species diversity and richness that could be sampled was captured by our sequencing depth (Fig. S17), indicated sufficient sequencing depth for majority of the analyzed samples. Estimated alpha diversity metrics using q2-diversity. Beta diversity metrics (Aitchison distance) and biplot were generated using DEICODE (robust Aitchison PCA, RPCA) [33]. Group significance between alpha and beta diversity indexes was calculated with QIIME2 plugins using the Kruskal-Wallis test and permutational multivariate analysis of variance (PERMANOVA), respectively. To assign taxonomy to ASVs, the q2-feature-classifier basing on the classify-sklearn naïve Bayes taxonomy classifier was used with the SILVA (v.138) as reference database. Unless specified (Fig. 2C, Fig.6B, Fig. S1A, Fig. S7), ASVs were grouped to the lowest classified taxonomy level (i.e., grouping $16 \mathrm{~S}$ sequencing at a specified taxonomic level, excluding those classified at lower levels) for all data modeling and analysis. The specified taxonomic levels are labelled with prefix "s_" (species level), "g_" (genus level), "f_" (family level), “o_" (order level), “c_" (class level), "p_" (phylum level), and "k_" (kingdom level) to indicate the taxonomic rank where grouping was operated. For example, "g_Bacteroides" clusters all sequences that are classified as Bacteroides at the genus level but unclassified at the species level. Alternatively, ASV sequences were grouped into Operational Taxonomic Units (OTUs) at 97\% similarity (Fig. S7).

For metagenome analysis, raw sequencing reads were subjected to quality filtering and barcode trimming using KneadData (v0.5.4) by employing trimmomatic settings of 4-base wide sliding window, with average quality per base $>20$ and minimum length $90 \mathrm{bp}$. Reads mapping to the mouse genome were removed. Kraken2 was run against genome taxonomy database (GTDB_r89_54k) with default parameters [72]. Following classification by Kraken2, Bracken was used to re-estimate bacterial abundances at taxonomic levels from species to phylum using a read length parameter of 150 . Next, the filtered sequences were assembled into contigs using metaSPAdes with default settings [73]. The gene abundance was analyzed and calculated as previously described with modifications [74]. Putative genes were then predicted

530 on contigs longer than 200 base pairs using Prodigal under metagenome mode (-p meta) [75]. A non-

531 redundant gene catalogue was constructed with CD-HIT using the parameters “-c 0.95 -aS 0.9" [76]. The

532 abundance of each predicted gene was evaluated by mapping reads back with KMA algorithm and then

533 normalized with the following equation: $\mathrm{RPM}=1 \mathrm{M} \times$ (mapped reads/gene length)/(sum of mapped

534 reads/gene length) [77]. For all the predicted genes, CAZymes were annotated using hmmsearch against 
the dbCAN2 database V9 (e value $<1 \times 10-10$; coverage $>0.3$ ) [78]. The domain with the highest coverage was selected for sequences overlapping multiple CAZyme domains. For all samples, short genomic assemblies $(<2,000 \mathrm{bp})$ that could have biased the subsequent analysis were first excluded. Genomes were then binned using VAMB [79]. The binning results were refined based on the bin quality assessment (completeness $>75$, and contamination $<15$ ) of different binners from CheckM [80]. Taxonomic classification of each bin was determined by GTDB-tk [81], and subjected to prediction of polysaccharide utilization loci (PULz) using pipeline PULpy [82].

Significance test of baseline-dependent responses. Sequential non-negative matrix factorization [38] was applied to transform all high-dimensional time series data from both intervention (inulin and resistant starch) and control group into two-dimensional space. We chose two factors because (1) reconstructed time series from the two latent factors preserve the quantitative trends of the untransformed time series sufficiently well and (2) two-dimensional data can be easily visualized. The reduced representation of the intervention group $\left\{\left(x_{v, i}, y_{v, i}\right)\right\}$ and control group $\left\{\left(p_{v, j}, q_{v, j}\right)\right\}, v(v=1,2, \ldots, V)$ refers to the index of vendor and $i, j(i=1,2, \ldots, N$ and $j=1,2, \ldots, N)$ refers to the index of mouse. For each vendor $v$, both vectors were then standardized by subtracting the mean vector of the vendor in the control group, i.e.,

$\left(x_{v, i}, y_{v, i}\right) \rightarrow\left(x_{v, i}^{\prime}=x_{v, i}-\frac{\sum_{k=1}^{N} p_{v, k}}{N}, y_{v, i}^{\prime}=y_{v, i}-\frac{\sum_{k=1}^{N} q_{v, k}}{N}\right)$ and $\left(p_{v, j}, q_{v, j}\right) \rightarrow\left(p_{v, j}^{\prime}=p_{v, j}-\right.$ $\left.\frac{\sum_{k=1}^{N} p_{v, k}}{N}, q_{v, j}^{\prime}=q_{v, j}-\frac{\sum_{k=1}^{N} q_{v, k}}{N}\right)$

The statistical significance test of: 1 ) the responsiveness (i.e., whether time series in the intervention group $\left(x_{v, i}^{\prime}, y_{v, i}^{\prime}\right)$ differs from that in the control group $\left(p_{v, j}^{\prime}, q_{v, j}^{\prime}\right)$ regardless of vendor), and 2$)$ the baseline dependence (i.e., whether time series in the intervention group $\left(x_{v, i}^{\prime}, y_{v, i}^{\prime}\right)$ varies among vendors $v$ ), were performed separately using Permutational Multivariate Analaysis of Variance (PERMANOVA) with Minkowski distance as the distance metric (the statistical module "statsmodels" in Python). We then obtained two P-values by comparing the differential responses between the intervention and control group ("responsiveness", Pr) as well as those between the four vendors in the intervention group ("baseline dependence”, $\mathrm{Pb}$ ). If both $\mathrm{P}$-valus are smaller than 0.05 , we determined that a quantity has a baselinedependent response. For all significance tests that require multiple test correction, the Benjamini-Hochberg procedure [83] was used for controlling the false discovery rate in multiple test correction.

564 Ecological inference of dietary fiber responses. The generalized Lotka-Volterra (gLV) model describes 565 how the absolute abundance of bacterial species change over time 


$$
\frac{d \log \left(x_{i}(t)\right)}{d t}=\alpha_{i}+\sum_{j=1}^{M} \beta_{i, j} x_{j}(t)+\epsilon_{i} u(t)
$$

567 where $M$ is the number of bacterial taxa, $x_{i}$ is the absolute abundance of taxon $i(i=1,2, \ldots, M), \alpha_{i}$ is the 568 basal growth rate, $\beta_{i, j}$ represents the influence of taxon $j(j=1,2, \ldots, M)$ on the growth of taxon $i, \epsilon_{i}$ is the susceptibility coefficient that represents growth response to dietary fiber, $u(t)$ is a binary variable that indicates whether the fiber is administed at time $t$. Bayesian regression techniques were used to parameterize the generalized Lotka-Volterra (gLV) model, as similarly used in Morjaria et al [39]. For each

572 mice $r(r=1,2, \ldots, P)$, Eq. (1) can be transformed into a matrix form that incorporates all discrete time 573 points of measurements $\left(t_{k}, k=1,2, \ldots, N\right)$

$$
\left[\begin{array}{c}
\left(\log \left(x_{1}\right)\right)_{t=t_{1}}^{\prime} \\
\vdots \\
\left(\log \left(x_{1}\right)\right)_{t=t_{N}}^{\prime} \\
\vdots \\
\left(\log \left(x_{M}\right)\right)_{t=t_{1}}^{\prime} \\
\vdots \\
\left(\log \left(x_{M}\right)\right)_{t=t_{N}}^{\prime}
\end{array}\right]=\left[\begin{array}{ccccccccccccccccccccccc}
1 & x_{1,1} & \cdots & x_{M, 1} & u_{1} & \cdots & 0 & 0 & \cdots & 0 & 0 & \cdots & 0 & 0 & \cdots & 0 & 0 & \cdots & 0 & 0 & \cdots & 0 & 0 \\
0 & 0 & \cdots & 0 & 0 & \cdots & 1 & x_{1, N} & \cdots & x_{M, N} & u_{N} & \cdots & 0 & 0 & \cdots & 0 & 0 & \cdots & 0 & 0 & \cdots & 0 & 0 \\
0 & 0 & \cdots & 0 & 0 & \cdots & 0 & 0 & \cdots & 0 & 0 & \cdots & 1 & x_{1,1} & \cdots & x_{M, 1} & u_{1} & \cdots & 0 & 0 & \cdots & 0 & 0 \\
0 & 0 & \cdots & 0 & 0 & \cdots & 0 & 0 & \cdots & 0 & 0 & \cdots & \vdots & 0 & \cdots & 0 & 0 & \cdots & 1 & x_{1, N} & \cdots & x_{M, N} & u_{N}
\end{array}\right]\left[\begin{array}{c}
\alpha_{1} \\
\beta_{1,1} \\
\vdots \\
\beta_{1, M} \\
\epsilon_{1} \\
\vdots \\
\alpha_{M} \\
\beta_{M, 1} \\
\vdots \\
\beta_{M, M} \\
\epsilon_{M} \\
\alpha_{M} \\
\beta_{M, 1} \\
\vdots \\
\beta_{M, M} \\
\epsilon_{M} \\
\vdots \\
\alpha_{M} \\
\beta_{M, 1} \\
\vdots \\
\beta_{M, M} \\
\epsilon_{M}
\end{array}\right]
$$

where $x_{i, k}=x_{i}\left(t_{k}\right)$ and $u_{k}=u\left(t_{k}\right)$. The log-derivatives of $x_{i}$ on the left-hand side of Eq. (2) were estimated from a cubic spline interpolation. Using a simplified notation for Eq. (2), i.e., $\mathbf{Y}_{\boldsymbol{r}}=\mathbf{X}_{\boldsymbol{r}} \mathbf{C}_{\boldsymbol{r}}$, we can incorpates data from all mice into a single regression model

$$
\left[\begin{array}{c}
\mathbf{Y}_{1} \\
\vdots \\
\mathbf{Y}_{P}
\end{array}\right]=\left[\begin{array}{ccc}
\mathbf{X}_{r} & \mathbf{0} & \mathbf{0} \\
\cdots & \cdots & \cdots \\
\mathbf{0} & \mathbf{0} & \mathbf{X}_{P}
\end{array}\right]\left[\begin{array}{c}
\mathbf{C}_{1} \\
\vdots \\
\mathbf{C}_{P}
\end{array}\right]
$$

The linear regression as described in Eq. (4) (for brevity $\mathbf{Y}=\mathbf{X C}$ ) can be further transformed into a Bayesian regression $\mathbf{Y}=\mathcal{N}(\mathbf{X C}, \sigma)$ where $\mathcal{N}$ and $\sigma$ represent normal distribution and standard deviation respectively. 
Since gLV models the absolute abundance of bacterial taxa, we multiplied the bacterial load by their relative abundance to calculate absolute abundance. The time-series data from all mice were simultaneously fed into the gLV model based on the premise that ecological forces driving microbiome dynamics are largely host-independent and universal [84]. We used uninformative Normal priors $\mathcal{N}(0,1)$ for all gLV parameters and Stan program [85] to produce posterior distributions for each parameter after "no U-turn" sampling of 10,000 samples from at least 3 independent Markov chain Monte Carlo traces. Since Stan is computationally expensive, we limited the inferences of dietary fiber responders to the top 20 bacterial taxa with the highest time-averaged absolute abundances in the inulin (or resistant starch) and cellulose group. Our Bayesian approach is conceptually similar to the Bayesian adaptive lasso algorithm in MDSINE [50]; the key difference is that MDSINE uses a hierarchical probability model and regularization to sample the variance parameters in the Normal priors.

Random forest (RF) model. Model development was run in a pipeline by combining normalization for data transformation, LASSO (least absolute shrinkage and selection operator) for feature selection, and RF regression for data fitting and prediction. The tolerance used in LASSO is 1e-5 and features whose coefficients below this threshold were discarded and not used to build RF regression model. Two data-split approaches were implemented. For the "interpolation" approach, the mice from the same vendor were first alphabetically labeled as A-D (for Hunan and Guangdong) or A-E (for Beijing and Shanghai). Then the four mice with the same label (one per vendor) were chosen to constitute the test set and the remaining mice were mixed together to form the training set. For the "extrapolation" approach, all mice from a specific vendor were chosen as the test set while the training set includes all mice from the other three vendors. To train each model, five hyperparameters were tuned using 5-fold cross validation within the training set and $\mathrm{R}^{2}$ as the scoring metric (GridSearchCV function of the scikit-learn library in Python): constant that multiplies the L1 term in LASSO (1e-4, 1e-3, 1e-2, 1e-1, 1), the number of features to consider when looking for the best split in RF (square root, $\log 2,16 \%, 32 \%, 64 \%, 100 \%$ of all features), the maximum depth of the tree in $\mathrm{RF}(2,4,8,16)$, the minium number of samples required to split an internal node in $\mathrm{RF}$ $(2,4,8,16)$, and the minimum number of samples required to be at a leaf node $(1,2,4)$. We fixed the number of trees in RF model to 2,000.

\section{Data availability}

613 All data (including metadata, sequencing data and metablomics data) have been deposited in the NCBI 614 SRA under accession number PRJNA754019.

\section{Code availability}


617 The customized scripts used in this study are available at: https://github.com/liaochen1988/DFdynamics.

618

\section{Acknowledgments}

620 We would like to thank members of LD lab for constructive comments on the manuscript. This research 621 was supported by National Key R\&D Program of China (No.2019YFA0906700, to L.D.), National Natural 622 Science Foundation of China (No.31971513, No. 32061143023, to L.D.) and China Postdoctoral Science 623 Foundation (2020M682968, to H.L.).

624

\section{Author Contributions}

626 H.L. and L.D. conceived the study. H.L. performed the experiments and analyzed the data. C. Liao analyzed 627 the sequencing data and performed the computational analysis. J.T., J.C., C. Lei, L.Z. and L.W. assisted in 628 experiments and/or data analysis. C. Liao, H.L. and L.D. wrote the manuscript with contributions from all 629 coauthors. 


\section{References:}

[1]. Tolhurst, G., et al., Short-chain fatty acids stimulate glucagon-like peptide-1 secretion via the Gprotein-coupled receptor FFAR2. Diabetes, 2012. 61(2): p. 364-71.

[2]. Vinolo, M.A.R., et al., Regulation of Inflammation by Short Chain Fatty Acids. Nutrients, 2011. 3(12): p. 858-876.

[3]. Litvak, Y., M.X. Byndloss and A.J. Bäumler, Colonocyte metabolism shapes the gut microbiota. Science, 2018. 362(6418): p. eaat9076.

[4]. Sanna, S., et al., Causal relationships among the gut microbiome, short-chain fatty acids and metabolic diseases. Nature Genetics, 2019. 51(4): p. 600-605.

[5]. Parada Venegas, D., et al., Short Chain Fatty Acids (SCFAs)-Mediated Gut Epithelial and Immune Regulation and Its Relevance for Inflammatory Bowel Diseases. Frontiers in Immunology, 2019. 10.

[6]. Zhao, L., et al., Gut bacteria selectively promoted by dietary fibers alleviate type 2 diabetes. Science, 2018. 359(6380): p. 1151-1156.

[7]. Sitkin, S., T. Vakhitov and J. Pokrotnieks, How to Increase the Butyrate-producing Capacity of the Gut Microbiome: Do IBD Patients Really Need Butyrate Replacement and Butyrogenic Therapy? Journal of Crohn's and Colitis, 2018. 12(7): p. 881-882.

[8]. Lordan, C., et al., Potential for enriching next-generation health-promoting gut bacteria through prebiotics and other dietary components. Gut microbes, 2019: p. 1-20.

[9]. David, L.A., et al., Diet rapidly and reproducibly alters the human gut microbiome. Nature, 2014. 505(7484): p. 559-63. [10]. Singh, V., et al., Microbiota fermentation-NLRP3 axis shapes the impact of dietary fibres on intestinal inflammation. Gut, 2019: p. gutjnl-2018-316250.

[11]. Healey, G., et al., Habitual dietary fibre intake influences gut microbiota response to an inulin-type fructan prebiotic: a randomised, double-blind, placebo-controlled, cross-over, human intervention study. British Journal of Nutrition, 2018. 119(2): p. 176-189.

[12]. Baxter, N.T., et al., Dynamics of Human Gut Microbiota and Short-Chain Fatty Acids in Response to Dietary Interventions with Three Fermentable Fibers. MBio, 2019. 10(1).

[13]. Deehan, E.C., et al., Precision Microbiome Modulation with Discrete Dietary Fiber Structures Directs Short-Chain Fatty Acid Production. Cell Host \& Microbe, 2020.

[14]. Venkataraman, A., et al., Variable responses of human microbiomes to dietary supplementation with resistant starch. Microbiome, 2016. 4(1): p. 33.

[15]. Nguyen, N.K., et al., Gut microbiota modulation with long-chain corn bran arabinoxylan in adults with overweight and obesity is linked to an individualized temporal increase in fecal propionate. Microbiome, 2020. 8(1).

[16]. Ze, X., et al., Ruminococcus bromii is a keystone species for the degradation of resistant starch in the human colon. ISME J, 2012. 6(8): p. 1535-43.

[17]. Lahti, L., et al., Tipping elements in the human intestinal ecosystem. Nat Commun, 2014. 5: p. 4344.

[18]. Rodriguez, J., et al., Discovery of the gut microbial signature driving the efficacy of prebiotic intervention in obese patients. Gut, 2020: p. gutjnl-2019-319726.

[19]. Kovatcheva-Datchary, P., et al., Dietary Fiber-Induced Improvement in Glucose Metabolism Is Associated with Increased Abundance of Prevotella. Cell Metabolism, 2015. 22(6): p. 971-982.

[20]. Coyte, K.Z., J. Schluter and K.R. Foster, The ecology of the microbiome: Networks, competition, and stability. Science, 2015. 350(6261): p. 663-6.

[21]. Davis, L.M.G., et al., Barcoded pyrosequencing reveals that consumption of galactooligosaccharides results in a highly specific bifidogenic response in humans. PloS one, 2011. 6(9): p. e25200.

ecosystem. Nature Microbiology, 2018. 3(11): p. 1274-1284.

680 [23]. Rakoff-Nahoum, S., M.J. Coyne and L.E. Comstock, An Ecological Network of Polysaccharide 681 Utilization among Human Intestinal Symbionts. Current Biology, 2014. 24(1): p. 40-49. 
682

683

684

685

686

687

688

689

690

691

692

693

694

695

696

697

698

699

700

701

702

703

704

705

706

707

708

709

710

711

712

713

714

715

716

717

718

719

720

721

722

723

724

725

726

727

728

729

730

731

732

[24]. Rao, C., et al., Multi-kingdom ecological drivers of microbiota assembly in preterm infants. Nature, 2021. 591(7851): p. 633-638.

[25]. Koskella, B., L.J. Hall and C. Metcalf, The microbiome beyond the horizon of ecological and evolutionary theory. Nat Ecol Evol, 2017. 1(11): p. 1606-1615.

[26]. Goldford, J.E., et al., Emergent simplicity in microbial community assembly. Science, 2018. 361(6401): p. 469-474.

[27]. Ortiz, A., et al., Interspecies bacterial competition regulates community assembly in the C. elegans intestine. ISME J, 2021. 15(7): p. 2131-2145.

[28]. Liu, Z., et al., Microbiome-based stratification to guide dietary interventions to improve human health. Nutrition Research, 2020. 82: p. 1-10.

[29]. Ahmed, W. and S. Rashid, Functional and therapeutic potential of inulin: A comprehensive review. Crit Rev Food Sci Nutr, 2019. 59(1): p. 1-13.

[30]. Cerqueira, F.M., et al., Starch Digestion by Gut Bacteria: Crowdsourcing for Carbs. Trends in Microbiology, 2019.

[31]. Parker, K.D., et al., Microbiome Composition in Both Wild-Type and Disease Model Mice Is Heavily Influenced by Mouse Facility. Frontiers in Microbiology, 2018. 9.

[32]. Ericsson, A.C., et al., Effects of Vendor and Genetic Background on the Composition of the Fecal Microbiota of Inbred Mice. PLOS ONE, 2015. 10(2): p. e0116704.

[33]. Martino, C., et al., A Novel Sparse Compositional Technique Reveals Microbial Perturbations. mSystems, 2019. 4(1).

[34]. Lagkouvardos, I., et al., Sequence and cultivation study of Muribaculaceae reveals novel species, host preference, and functional potential of this yet undescribed family. Microbiome, 2019. 7(1).

[35]. Pereira, F.C., et al., Rational design of a microbial consortium of mucosal sugar utilizers reduces Clostridiodes difficile colonization. Nat Commun, 2020. 11(1): p. 5104.

[36]. Wu, G.D., et al., Linking long-term dietary patterns with gut microbial enterotypes. Science, 2011. 334(6052): p. 105-8.

[37]. Creswell, R., et al., High-resolution temporal profiling of the human gut microbiome reveals consistent and cascading alterations in response to dietary glycans. Genome Medicine, 2020. 12(1).

[38]. Mackevicius, E.L., et al., Unsupervised discovery of temporal sequences in high-dimensional datasets, with applications to neuroscience. Elife, 2019. 8.

[39]. Morjaria, S., et al., Antibiotic-Induced Shifts in Fecal Microbiota Density and Composition during Hematopoietic Stem Cell Transplantation. Infect Immun, 2019. 87(9).

[40]. Stein, R.R., et al., Ecological modeling from time-series inference: insight into dynamics and stability of intestinal microbiota. PLoS Comput Biol, 2013. 9(12): p. e1003388.

[41]. Rakoff-Nahoum, S., K.R. Foster and L.E. Comstock, The evolution of cooperation within the gut microbiota. Nature, 2016. 533(7602): p. 255-259.

[42]. Koropatkin, N.M., E.A. Cameron and E.C. Martens, How glycan metabolism shapes the human gut microbiota. Nat Rev Microbiol, 2012. 10(5): p. 323-35.

[43]. Chijiiwa, R., et al., Single-cell genomics of uncultured bacteria reveals dietary fiber responders in the mouse gut microbiota. Microbiome, 2020. 8(1): p. 5-14.

[44]. Zhou, K., Strategies to promote abundance of Akkermansia muciniphila, an emerging probiotics in the gut, evidence from dietary intervention studies. J Funct Foods, 2017. 33: p. 194-201.

[45]. Goldford, J.E., et al., Emergent simplicity in microbial community assembly. Science, 2018. 361(6401): p. 469-474.

[46]. Wu, G., et al., Guild-based analysis for understanding gut microbiome in human health and diseases. Genome Med, 2021. 13(1): p. 22.

[47]. Patnode, M.L., et al., Interspecies Competition Impacts Targeted Manipulation of Human Gut Bacteria by Fiber-Derived Glycans. Cell, 2019. 179(1): p. 59-73.e13.

[48]. Salonen, A., et al., Impact of diet and individual variation on intestinal microbiota composition and fermentation products in obese men. ISME J, 2014. 8(11): p. 2218-30.

[49]. Sze, M.A., et al., Fecal Short-Chain Fatty Acids Are Not Predictive of Colonic Tumor Status and 
Cannot Be Predicted Based on Bacterial Community Structure. mBio, 2019. 10(4): p. e01454-19.

[50]. Bucci, V., et al., MDSINE: Microbial Dynamical Systems INference Engine for microbiome timeseries analyses. Genome Biology, 2016. 17(1).

[51]. Buffie, C.G., et al., Precision microbiome reconstitution restores bile acid mediated resistance to Clostridium difficile. Nature, 2015. 517(7533): p. 205-8.

[52]. Lagkouvardos, I., et al., The Mouse Intestinal Bacterial Collection (miBC) provides host-specific insight into cultured diversity and functional potential of the gut microbiota. Nature Microbiology, 2016. $1(10)$.

741 [53]. Atkinson, G. and A.M. Batterham, True and false interindividual differences in the physiological response to an intervention. Experimental Physiology, 2015. 100(6): p. 577-588.

[54]. Schloss, P.D., Identifying and Overcoming Threats to Reproducibility, Replicability, Robustness, and Generalizability in Microbiome Research. mBio, 2018. 9(3).

[55]. Baxter, N.T., et al., The Glucoamylase Inhibitor Acarbose Has a Diet-Dependent and Reversible Effect on the Murine Gut Microbiome. mSphere, 2019. 4(1).

[56]. Walker, A.W., et al., Dominant and diet-responsive groups of bacteria within the human colonic microbiota. ISME J, 2011. 5(2): p. 220-30.

[57]. Hiel, S., et al., Effects of a diet based on inulin-rich vegetables on gut health and nutritional behavior in healthy humans. Am J Clin Nutr, 2019. 109(6): p. 1683-1695.

[58]. Nordgaard, I., et al., Colonic production of butyrate in patients with previous colonic cancer during long-term treatment with dietary fibre (Plantago ovata seeds). Scand J Gastroenterol, 1996. 31(10): p. 101120.

[59]. Sakata, T., Pitfalls in short-chain fatty acid research: A methodological review. Animal Science Journal, 2019. 90(1): p. 3-13.

[60]. Gurry, T., et al., Functional heterogeneity in the fermentation capabilities of the healthy human gut microbiota. PLoS One, 2021. 16(7): p. e0254004.

[61]. Johnson, A.J., et al., A Guide to Diet-Microbiome Study Design. Frontiers in Nutrition, 2020. 7.

[62]. Shepherd, E.S., et al., An exclusive metabolic niche enables strain engraftment in the gut microbiota. Nature, 2018. 557(7705): p. 434-438.

[63]. Kolodziejczyk, A.A., D. Zheng and E. Elinav, Diet-microbiota interactions and personalized nutrition. Nat Rev Microbiol, 2019. 17(12): p. 742-753.

[64]. Zhang, S., H. Wang and M. Zhu, A sensitive GC/MS detection method for analyzing microbial metabolites short chain fatty acids in fecal and serum samples. Talanta, 2019. 196: p. 249-254.

[65]. Cai, J., et al., Orthogonal Comparison of GC-MS and 1H NMR Spectroscopy for Short Chain Fatty Acid Quantitation. Analytical Chemistry, 2017. 89(15): p. 7900-7906.

[66]. Jian, C., et al., Quantitative PCR provides a simple and accessible method for quantitative microbiota profiling. PLOS ONE, 2020. 15(1): p. e0227285.

[67]. Gohl, D.M., et al., Systematic improvement of amplicon marker gene methods for increased accuracy in microbiome studies. Nature Biotechnology, 2016. 34(9): p. 942-949.

[68]. Klindworth, A., et al., Evaluation of general 16S ribosomal RNA gene PCR primers for classical and next-generation sequencing-based diversity studies. Nucleic Acids Research, 2013. 41(1): p. e1-e1.

[69]. Bolyen, E., et al., Reproducible, interactive, scalable and extensible microbiome data science using QIIME 2. Nat Biotechnol, 2019. 37(8): p. 852-857.

[70]. Davis, N.M., et al., Simple statistical identification and removal of contaminant sequences in marker-gene and metagenomics data. Microbiome, 2018. 6(1).

[71]. Hsieh, T.C., K.H. Ma and A. Chao, iNEXT: an R package for rarefaction and extrapolation of species diversity (H ill numbers). Methods in Ecology and Evolution, 2016. 7(12): p. 1451-1456.

[72]. Wood, D.E., J. Lu and B. Langmead, Improved metagenomic analysis with Kraken 2. Genome Biology, 2019. 20(1).

781 [73]. Nurk, S., et al., metaSPAdes: a new versatile metagenomic assembler. Genome Res, 2017. 27(5): 782 p. $824-834$.

783 [74]. Zhao, Z., F. Baltar and G.J. Herndl, Linking extracellular enzymes to phylogeny indicates a 
predominantly particle-associated lifestyle of deep-sea prokaryotes. Science advances, 2020. 6(16): p. eaaz4354.

786 [75]. Hyatt, D., et al., Prodigal: prokaryotic gene recognition and translation initiation site identification. BMC Bioinformatics, 2010. 11: p. 119.

788 [76]. Fu, L., et al., CD-HIT: accelerated for clustering the next-generation sequencing data. Bioinformatics, 2012. 28(23): p. 3150-2.

790 [77]. Clausen, P.T.L.C., F.M. Aarestrup and O. Lund, Rapid and precise alignment of raw reads against 791 redundant databases with KMA. BMC Bioinformatics, 2018. 19(1).

792 [78]. Zhang, H., et al., dbCAN2: a meta server for automated carbohydrate-active enzyme annotation. 793 Nucleic Acids Research, 2018. 46(W1): p. W95-W101.

794 [79]. Nissen, J.N., et al., Improved metagenome binning and assembly using deep variational 795 autoencoders. Nature Biotechnology, 2021.

796 [80]. Parks, D.H., et al., CheckM: assessing the quality of microbial genomes recovered from isolates, single cells, and metagenomes. Genome Res, 2015. 25(7): p. 1043-55.

799 Database. Bioinformatics, 2019.

800 [82]. Stewart, R.D., et al., Open prediction of polysaccharide utilisation loci (PUL) in 5414 public 801 Bacteroidetes genomes using PULpy. bioRxiv, 2018: p. 421024.

802 [83]. McDonald, J.H., Handbook of biological statistics. Vol. 2. 2009: sparky house publishing 803 Baltimore, MD.

804 [84]. Bashan, A., et al., Universality of human microbial dynamics. Nature, 2016. 534(7606): p. 259-

805262.

806 [85]. Carpenter, B., et al., Stan: a probabilistic programming language. Grantee Submission, 2017. 76(1): 807 p. 1-32. 